A total of $111(52.3 \%)$ babies were premature. The mean birth weight of the babies was 2052 \pm 352 gms (1050-2490 gms) and the mean gestational age was $35 \pm 2$ weeks (28-42 weeks).

Out of 212 babies, ninety-nine babies (46.7\%) had a low ponderal index $(<2.2$ in term babies, $<2.0$ in preterms $)$ and by definition had disproportionate IUGR, out of which 60 (59.4\%) babies were term and 39 (35.1\%) were preterms.

170 babies $(80.2 \%)$ had no postnatal complications, 15 babies had neonatal sepsis, 10 babies had respiratory distress syndrome, 6 babies had hypoxic-ischemic encephalopathy (4 babies stage I HIE, 2 babies Stage II HIE), 8 babies had transient tachypnea of newborn, 3 babies had hypoglycemia and 2 babies had polycythemia.

Conclusions Ponderal index is a simple and effective tool to identify wasting. In this study, nearly $46.7 \%$ of the low birth weight babies were disproportionate at birth.

\section{PARENTAL DECISION MAKING WHEN CHOOSING A PAEDIATRIC PRIMARY CARE SERVICE: WHAT FACTORS MATTERED MOST?}

Erwin Jiayuan Khoo, Colette Teng Wee, Kurubaran Ganasegeran. Malaysia

\subsection{6/bmjpo-2021-RCPCH.63}

Background In Malaysia, patients are free to choose their own primary care healthcare providers at their own expense. Studies show that patients who choose their own doctor are associated with increased patient satisfaction. Primary care paediatricians realise the need to involve parents as stakeholders in today's competitive healthcare industry.

Objectives This study aimed to identify what factors matter to parents when choosing a paediatric primary care for their children in a free market setting where they have direct access to paediatricians. It also explored if differences in these perceived factors exists between parental education and household income levels.

Methods An analytical cross-sectional study was conducted at two paediatric primary care centres in urban Malaysia. Parents who brought their child to seek treatment at the centres were invited to participate. A face and content validated questionnaire that includes respondents' demographics was devised from the literature and a focus group discussion. A 5-point Likert scale was applied to 25 elements that were organised into five major domains: 'consultations' (CO), 'facilities and services' (FS), 'fees and charges' (FC), 'social media engagement' (SM) and 'doctor's 'appearance' (DA). Elements within each domain were ranked according to a mean ratings score. Internal consistency for each domain was yielded through a Cronbach's alpha $(\alpha)$ value. Independent t-test was used to determine the associations between outcomes and independent variables.

Results 387 parents completed the questionnaires (response rate, 91.3\%). The mean age of the parents was 37.7 years. $295(76.2 \%)$ parents attained a tertiary education. 157 (40.6\%) parents were bottom tier income earners. Parents rated the provision of FS highest (mean rating score $=41.63$, $\alpha=0.84$ ), followed by ratings on the interaction with healthcare providers during $\mathrm{CO}(34.73,0.77)$. Younger aged parents $(p=0.021)$ and parents from lower household income level $(p=0.001)$ had higher FS rating score compared with older parents and parents of higher income respectively. The top elements in the FS domain were a 'strategic location of the primary care' and 'comfortable and clean waiting room'. The $\mathrm{CO}$ ratings have a predilection towards technical quality, interpersonal manners and successful provider-patient communications during clinical encounters. In exchange, for FC (11.80, $0.75)$, parents appeared to tolerate costlier consultation and treatment, but still appreciate bills that are transparent. While parents with a higher level of education prefer to participate in shared decision-making with the paediatricians $(p=0.002)$, parents from lower household income level rated a significantly higher score in SM $(p<0.001)$ and DA $(p=0.019)$.

Conclusions Where parents are free to choose their children's paediatric primary care service, convenient accessibility and perceived consultation-related proficiency mattered most to parents when deciding. The extent to what makes a difference, appeared to be influenced by parental age, educational and income level. These perceived differences largely confirm the need to invest in the development of individualised support services in a consumer-driven health care system. These preferred elements can provide clue to determine parental satisfaction towards health services provided and predict complex health seeking behaviours.

\section{ASSOCIATION BETWEEN DEGREE OF SENSITIVITY TO INDOOR ALLERGENS AND ASTHMA SEVERITY}

Wai Mar Tin. Myanmar

\subsection{6/bmjpo-2021-RCPCH.64}

Background Aeroallergens, especially indoor aeroallergens have a major role in the pathogenesis and severity of asthma. In Myanmar, data about allergen sensitivity and asthma is still lacking. Understanding the prevalence of the most common aeroallergens in local area can help in the diagnosis and management of asthma.

\section{Objectives}

1. To determine the prevalence of sensitivity of indoor allergens in children with asthma

2. To analyze the association between degree of sensitivity to indoor allergens and asthma severity.

Methods This hospital based cross sectional analytical study was conducted in 120 children (aged 6-12 years) with asthma who attended asthma clinic in Yangon Children Hospital. Severity of asthma was assessed by taking history, physical examination and lung function test. It was classified into mild asthma and moderate to severe asthma groups according to the working definition. Skin prick test with eight indoor aeroallergens extracts was performed in 120 children. The degree of the allergic sensitization was assessed by the number of positive skin prick tests and by skin wheal sizes. Associations between degree of atopic sensitization and severity of asthma, were analyzed by using logistic regression

Results The majority of subjects $(67.5 \%)$ had moderate- severe asthma and $32.5 \%$ had mild asthma. $92.5 \%$ of study population had skin prick test positive to at least one indoor allergen. Mono sensitization to aeroallergen was observed in only $11.7 \%$ of patients and the rest were sensitized to more than one allergen among sensitized children. The most prevalent allergen was HDM (Dermatophagoides pteronyssinus and Dermatophagoides farinae) with $64.2 \%$ and $68.3 \%$ respectively. Prevalence of sensitization to other indoor allergens was as follow: Aspergillus fumigatus (40.8\%), Alternaria alternata 
(34.2\%), Cat (51.7\%), Dog (50.8\%) and cockroaches, Blatella germanica (48.3 8\%) and Periplaneta Americana (53.3\%). Overall, multiple sensitization (sensitization to $>4$ allergens) was significantly associated with increasing level of asthma severity $(\mathrm{p}<0.001)$. However, that association disappeared after adjustment for potential confounders like age, sex, exposure to smoking, pet and having family history of asthma or allergic rhinitis. It remained statistically significant only for the patients sensitized to HDM as the relationship was not modified by con founders. Moreover, mean SPT wheal size was not significantly associated with severity of asthma for all allergens tested.

Conclusions HDM was the most common sensitizing aeroallergen. Although children with moderate to severe asthma had significantly higher proportion of SPT positive, this study could not establish any relationship between degree of wheal size and severity of asthma. Therefore, the association between degree of sensitivity to indoor allergen and childhood asthma severity is still uncertain.

\section{DIAGNOSTIC DIFFICULTY DURING COVID 19 PANDEMIC- SICKLE CELL CRISIS OR PIMS-TS?}

Kyla Lief, Maggie Nyirenda. UK

10.1136/bmjpo-2021-RCPCH.65

Background The following case report highlights the difficulty in distinguishing Paediatric Multisystem Inflammatory Syndrome Temporally Associated with Sars- CoV- 2 (PIMS -TS) from sickle cell crisis. This is important in the context of a looming second wave of the Covid 19 pandemic, particularly in areas where there is a large population of children with sickle cell disease. This is the first case report discussing PIMS TS in a child with sickle cell disease and pulmonary emboli as a result of this.

Objectives A 17 year old boy with sickle cell disease presented to the paediatric department of a district general hospital in South London with pleuritic chest pain and worsening shortness of breath in April 2020. He was tachypnoeic and tachycardic. Oxygen saturation was $95 \%$ in air and he was afebrile. He had quiet breath sounds and was tender in the right upper quadrant of his abdomen. A week prior to this he required intravenous (IV) antibiotics and two exchange transfusions via a femoral line. Blood tests showed a white cell count (WCC) of 11.4 , a C-reactive protein (CRP) of 120, haemoglobin of 108 , INR of 1.6 and deranged liver function tests. His D dimer was 8339 , so a pulmonary Computed Tomography (CT) angiogram was performed. This demonstrated bilateral pulmonary emboli (PE).

Methods

Results Differential diagnoses for the cause of the PE were sickle cell disease and PIMS-TS.

Conclusions Both PIMS -TS and sickle cell crises are disorders involving exaggerated inflammation and risk of coagulopathy, with raised CRP, D Dimer, INR and WCC. The guidelines for investigations of PIMS TS include other markers such as LDH, troponin, BNP, ferritin and creatinine kinase. However, these blood tests are rarely performed in the general paediatric population or those with sickle cell disease, so they need to be specifically studied to determine whether they provide any significant distinction for a diagnosis of PIMS-TS.
In terms of pathophysiology, a diagnosis of $\mathrm{PE}$ as a complication of sickle cell is not surprising; however in clinical practice it is rarely seen. This points towards PIMS-TS being the cause of this presentation, for which WHO definition requires evidence of SARSCOV2 infection or exposure. Indeed our patient is likely to have been exposed to Covid 19, given that he presented in the peak of the first wave, however PCR swabs were negative during admission. Three months after presentation, his anti Sars Cov $2 \mathrm{IgG}$ was negative.

The key take home message from this case is to consider other diagnoses in sickle cell patients during the time of Covid 19. There is a need for increased research into how to differentiate the two disorders. This is important because if the primary cause of illness is PIMS-TS rather than sickle cell disease, careful consideration needs to go into treatments as immunomodulation with IV Ig may increase viscosity and steroids can contribute to hypertension, thus worsening the progression of underlying sickle cell disease. Diagnosing PIMS TS in those with sickle cell is particularly important as patients from a minority ethnic suffer worse outcomes.

\section{ENHANCING LEARNING AND IMPROVING FEEDBACK THROUGH QR CODES}

Kee Wei Phang, Poonam Patel. UK

\subsection{6/bmjpo-2021-RCPCH.66}

Background North Middlesex University Hospital is a District General Hospital based in Central London and has recently developed a weekly ED simulation programme as part of specialty training. The simulation programme is run by a simulation fellow and ED consultant and aims to improve trainees' confidence in core ED competencies including trauma and paediatric emergencies. It is recognised that there are difficulties in obtaining accurate and complete feedback from participants due to time constraints i.e. need to return to clinical work; because traditional methods of collecting feedback via paper forms are cumbersome and has an environmental impact with paper wastage, or feedback is lost through illegible writing. To improve the collection of feedback, we created a standardised online feedback form which participants access via a $\mathrm{QR}$ code using their mobile phones, and can be filled in quickly and accurately. This process will soon be rolled out to the entire hospital as the standardised method of collecting feedback from simulation sessions.

\section{Objectives}

1. To improve the collection of feedback after simulation sessions, such that the simulation team is better able to capture feedback from all participants and analyse a complete set of data.

2. To improve data analysis with electronic record by reducing human errors in transcribing feedback from paper forms during analysis.

3. To standardise the feedback collection process in the hospital.

4. Reduce paper wastage and become more environmentally friendly.

Methods Participants scan a QR code that is made available at the start and end of the simulation sessions which directs them to a short online feedback form where they can fill in feedback anonymously.

Results There is an average of $83 \%$ feedback response rate from the simulation sessions conducted between Sept 2020 to 